\title{
Scaling to generalize a single solution of Richards' equation for soil water redistribution
}

\author{
Morteza Sadeghi ${ }^{1 *}$, Bijan Ghahraman ${ }^{1}$, Kamran Davary ${ }^{1}$, Seyed Majid Hasheminia ${ }^{1}$, Klaus \\ Reichardt ${ }^{2}$ \\ ${ }^{1}$ Ferdowsi University of Mashbad/College of Agriculture - Dept. of Water Engineering - 91779-4897 - Mashbad, Iran. \\ ${ }^{2}$ USP/CENA - Lab. de Física do Solo, C.P. 96 - 13418-900 - Piracicaba, SP - Brasil. \\ *Corresponding author <m.sadeghi.um@gmail.com> \\ Edited by: Jussara Borges Regitano
}

\begin{abstract}
Using scaling methods, a single solution of Richards' equation (RE) will suffice for numerous specific cases of water flow in unsaturated soils. In this study, a new method is developed to scale RE for the soil water redistribution process. Two similarity conditions are required: similarity in the shape of the soil water content profiles as well as of the water flux density curves. An advantage of this method is that it is not restricted to a specific soil hydraulic model - hence, all such models can be applied to RE. To evaluate the proposed method, various soil textures and initial conditions were considered. After the RE was solved numerically using the HYDRUS-1D model, the solutions were scaled. The scaled soil water content profiles were nearly invariant for medium- and fine-textured soils when the soil profile was not deeply wetted. The textural range of the soils in which the similarity conditions are held decreases as the initial conditions deal with a deeply wetted profile. Thus, the scaling performance was poor in such a condition. This limitation was more pronounced in the coarse-textured soils. Based on the scaling method, a procedure is suggested by which the solution of RE for a specific case can be used to approximate solutions for many other cases. Such a procedure reduces complicated numerical calculations and provides additional opportunities for solving the highly nonlinear RE as in the case of unsaturated water flow in soils.
\end{abstract}

Keywords: Nielsen-similarity, spatial variability, invariant solutions

\section{Introduction}

Scaling methods based on the "similar media" concept (Miller and Miller, 1956) were developed to cope with the spatial variability of soils (Warrick et al., 1977; Sharma et al., 1980; Ahuja and Williams, 1991; Kosugi and Hopmans, 1998; Tuli et al., 2001; Kozak and Ahuja, 2005; Roth, 2008; Sadeghi et al., 2010). Vereecken et al. (2007) comprehensively reviewed the scaling methods developed during the past years.

Scaling has proven its success also as a tool for numerical analyses. Using scaling methods, a single solution of Richards' equation (RE) will suffice for numerous specific cases of unsaturated water flow. Hence, these methods considerably reduce the calculations required for heterogeneous soils (Warrick and Hussen, 1993). So far, various methods for scaling RE have been proposed (Reichardt et al., 1972; Warrick and AmoozegarFard, 1979; Warrick et al., 1985; Vogel et al., 1991; Kutilek et al., 1991; Warrick and Hussen, 1993; Neuweiler and Cirpka, 2005). Using specific scaling factors, these methods allow a linear transformation of RE variables to achieve invariant solutions for a set of similar soils. This similarity may be defined based on microscopic-scale geometry (Miller and Miller 1956), shape of soil hydraulic functions (Simmons et al., 1979), or a linear variability concept (Vogel et al., 1991).

Scaling methods can be divided into two classes (Kutilek and Nielsen, 1994). In the first class, the scaling factors are derived to unify the soil hydraulic functions into a single curve and therefore, are invariant quantities for each soil. Using these methods, scaled RE will be invariant for similar soils provided that the scaled boundary and initial conditions are the same. This provision limits the applicability of such methods developed, for example, by Warrick et al. (1985), and Vogel et al. (1991). In the second class, the scaling factors are defined by considering the imposed boundary and/or initial conditions. A clear advantage of these methods over the first class is that the scaled RE is invariant to the boundary and/or initial conditions. However, these methods are limited to specific hydrological processes and/or soil hydraulic models. For example, the method of Warrick and Hussen (1993), developed for infiltration and redistribution, applies only for the Brooks-Corey hydraulic functions.

The objective of this study, following the second class, is to scale RE focused on the redistribution process. Scaling of $\mathrm{RE}$ solutions is proposed instead of solving the scaled RE. By this procedure, this new method is not restricted to a specific hydraulic model - hence, all existing and future models can be used for the redistribution solutions.

\section{Theory}

Consider a one-dimensional Richards' equation (RE) of the form:

$$
\frac{\partial \theta}{\partial t}=-\frac{\partial}{\partial z}\left(K \frac{\partial b}{\partial z}+K\right)
$$

where $\theta\left[\mathrm{L}^{3} \mathrm{~L}^{-3}\right]$ is the volumetric soil water content, $K\left[\mathrm{~L} \mathrm{~T}^{-1}\right]$ the unsaturated hydraulic conductivity, $b[\mathrm{~L}]$ the soil water matric potential head (i.e. absolute value of the soil water pressure head), $z$ the vertical position coordinate below soil surface, and $t$ the time.

Considering a soil water redistribution process without surface evaporation, the following conditions are imposed on the upper $(z=0)$ and lower boundary $(z=L)$ of the solution 
domain dealing with zero water flux density and free drainage, respectively:

$q(0, t)=0$

$q(L, t)=K(L, t)$

where $q\left[\mathrm{~L} \mathrm{~T}^{-1}\right]$ is the water flux density. The following initial conditions are considered:

$$
\begin{array}{ll}
\theta(z, 0)=\theta_{f_{i}} & \left(0<z<z_{f i}\right) \\
\theta(z, 0)=\theta_{i} & \left(z>z_{f i}\right)
\end{array}
$$

where $\theta_{f_{i}}$ and $z_{f_{i}}$ are the initial values of the wetting front water content $\theta_{f}(t)$ and depth $z_{f}(t)$, respectively; $\theta_{i}$ is the initial soil water content below the wetting front (WF). Figure 1 graphically describes the imposed boundary and initial conditions on the solution domain.

A $\theta$-based solution of Eq. (1), considering conditions (2) to (4), yields soil water content profiles (SWCP), $\theta(z)$, during redistribution. Considering the shape of the SWCPs during redistribution, two general forms can be found: the SWCP (a) with the $\theta$ gradient everywhere positive and with the $\theta$ distribution almost uniform above a sharp WF, and (b) with a further wetting below advancing as a step-like profile while the soil desaturates near the surface (Youngs, 1990).

\section{Scaling Method}

Following relationships are proposed to scale $\theta$ and $z$ :

$$
\begin{aligned}
& \theta^{*}=\frac{\theta-\theta_{i}}{\theta_{f i}-\theta_{i}} \\
& z^{*}=\frac{Z}{z_{f i}}
\end{aligned}
$$

by which the scaled values of $\theta_{f i}$ and $z_{f i}$ will become unity and the scaled initial conditions will be invariant as follows:

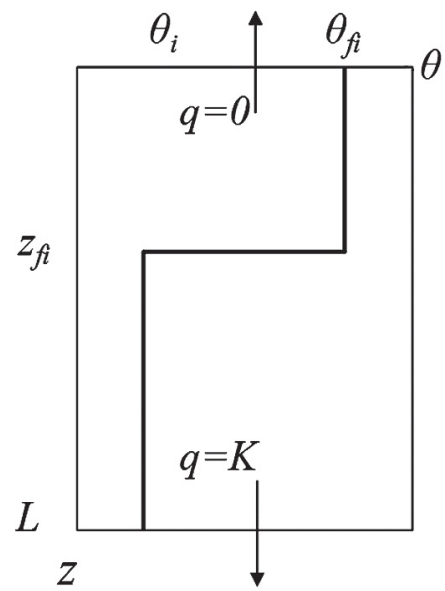

Figure 1 - Graphical description of the imposed boundary and initial conditions on the soil profile during redistribution process.

$$
\begin{aligned}
& \theta^{*}\left(z^{*}, t=0\right)=1 \quad\left(0<z^{*}<1\right) \\
& \theta^{*}\left(z^{*}, t=0\right)=0 \quad\left(z^{*}>1\right)
\end{aligned}
$$

In Eqs. (5) to (7) $\theta^{*}$ and $z^{*}$ are the scaled soil water content and depth, respectively. It is assumed that $t$ can be scaled using a constant scaling factor $\tau$ (i.e. $t^{*}=\tau t$, where $t^{*}$ is the scaled time) so that the scaled solutions of RE, $\theta^{*}\left(z^{*}, t^{*}\right)$, be invariant for a set of cases (i.e. specific soils and initial conditions). To do so, a primary requirement is that the shape of the SWCPs be similar for all the cases. Assume two cases $A$ and $B$ for which this condition is held. Since the scaled initial conditions are invariant, the scaled solutions will be the same for these two cases only if the scaled WF advance velocity (i.e. $d z_{f}^{*} / d t^{*}$, where $z_{f}^{*}$ is the scaled WF depth) is the same at each scaled time:

$$
\left[\frac{d z_{f}^{*}}{d t^{*}}\left(t^{*}\right)\right]_{A}=\left[\frac{d z_{f}^{*}}{d t^{*}}\left(t^{*}\right)\right]_{B}
$$

where subscripts $A$ and $B$ correspond to cases $A$ and $B$. Eq. (8) gives:

$$
\frac{\left[v_{f}\left(t^{*}\right)\right]_{A}}{\left[v_{f}\left(t^{*}\right)\right]_{B}}=\frac{\tau_{A}}{\tau_{B}}=\text { cons. }=\frac{\left(v_{f i}\right)_{A}}{\left(v_{f i}\right)_{B}}
$$

here $v_{f}\left[\mathrm{~T}^{-1}\right]$ represents $d z_{f}^{*} / d t$, and $v_{f}$ is the initial value of $v_{f}$ Based on Eq. (9), $v_{f i}$ will be the best choice for the time scaling factor, $\tau$ :

$t^{*}=v_{f_{t}} t$

To determine $v_{f i}$, Darcy's equation is considered in the form of:

$q=K \frac{\partial b}{\partial z}+K$

Integrating Eq. (11) from the soil surface to the WF depth at $t$ $=0$, we have:

$\int_{0}^{z_{f i}}(q-K) d z=\int_{h_{f i}}^{b_{i}} K d h$

which yields:

$q_{f i}=\frac{K_{s} G}{z_{f i}}+K_{f i}$

In Eqs. (12) and (13), $h_{i}[\mathrm{~L}]$ is the matric potential head corresponding to $\theta_{i}, h_{f_{i}}[\mathrm{~L}]$ and $K_{f_{i}}\left[\mathrm{LT}^{-1}\right]$ are the matric potential head and hydraulic conductivity corresponding to $\theta_{f_{i}}$, respectively, $\theta_{f_{i}}$ is the initial value of downward flux density at the WF, $K_{s}\left[\mathrm{LT}^{-1}\right]$ is the saturated hydraulic 
conductivity, and $G[\mathrm{~L}]$ is WF matric head at $t=0$ defined as:

$$
G=\frac{1}{K_{s}} \int_{h_{f i}}^{h_{i}} K d h
$$

Mass conservation law gives:

$$
q_{f i}=\left(\theta_{f i}-\theta_{i}\right)\left(\frac{d z_{f}}{d t}\right)_{t=0}=\left(\theta_{f i}-\theta_{i}\right) z_{f i} v_{f i}
$$

Combining (13) and (15) results in:

$v_{f i}=\frac{K_{s} G}{\left(\theta_{f i}-\theta_{i}\right) z_{f i}^{2}}+\frac{K_{f i}}{\left(\theta_{f i}-\theta_{i}\right) z_{f i}}$

Using Eq. (16), $v_{f i}$ can be calculated from the initial conditions (i.e. $\theta_{f i}, \theta_{i}$ and $z_{f i}$ ). The soil hydraulic functions should be known to determine $K_{f i}$ and $h_{f i}$ from $\theta_{f i}$ as well as $b_{i}$ from $\theta_{i}\left(b_{f i}\right.$ and $h_{i}$ are required to calculate $G$ using Eq. (14)). Although there is no restriction on a specific form of the soil hydraulic models, it should be noticed that the selected hydraulic models should be the same for solving RE and calculating $v_{f i}$ using Eq. (16). Based on the mass conservation law, $\partial \theta / \partial t=-\partial q / \partial z$, Eqs. (5), (6), and (10) suggest the following relationship to scale the flux density:

$q^{*}\left(z^{*}\right)=\frac{q(z)}{\left(\theta_{f i}-\theta_{i}\right) z_{f i} v_{f i}}=\frac{q(z)}{q_{f i}}$

where $q^{*}$ is the scaled flux density. To scale the redistribution process for a set of soils and initial conditions, $q^{*}\left(z^{*}\right)$ should be a unified curve. Eq. (17) implies in another condition necessary for the scaling, dealing with the shape similarity of the flux density curves over the scaled soil profile, such that it can be scaled by a linear transformation. This kind of similarity was previously adopted by Simmons et al. (1979) and, as stated by Sposito and Jury (1985), is referred to as "Nielsen-similarity".

\section{Materials and Methods}

Richards' Equation was solved using HYDRUS-1D, Version 4 (Simunek et al., 2008) for various soils and initial conditions. To simulate the SWCPs during redistribution, zero water flux and free drainage were set as the upper and lower boundary conditions, respectively. Van Genuchten (1980) hydraulic functions based on Mualem's (1976) model were adopted:

$\theta=\theta_{r}+\frac{\left(\theta_{s}-\theta_{r}\right)}{\left[1+(\alpha b)^{n}\right]^{m}}$
$K=K_{s} \frac{\left\{1-(\alpha b)^{n-1}\left[1+(\alpha h)^{n}\right]^{-m}\right\}^{2}}{\left[1+(\alpha b)^{n}\right]^{m / 2}}$

where $\theta_{r}$ and $\theta_{s}$ are soil residual and saturated water contents, respectively, and $\alpha, n$, and $m$ are empirical parameters with the assumption that $m=1-1 / n$.

Soils of twelve textural classes were considered by applying parameters of Carsel and Parrish (1988) which are the default parameters of HYDRUS for van Genuchten functions (Table 1). Two sets of initial conditions, presented in Table 2, were considered using various combinations of $z_{f_{i}}, h_{f_{i}}$ and $h_{i}$. Set A (A1 to A6) was considered to evaluate the effect of the total water added to the soil profile, $W$, while Set B (B1 to B8) was considered to separately evaluate the effects of $z_{f i}, h_{f i}$ and $h_{i}$. To prevent the divergence of the solutions, the abrupt increase of the matric potential head at the WF was avoided. To do so, the matric potential head gradually increased from $b_{f i}$ and $b_{i}$ through four space steps at the WF. It should be noted that HYDRUS works with the pressure head (negative values) instead of the absolute value of the matric head.

SWCPs obtained by HYDRUS were scaled using Eqs. (5), (6), and (10). The value of $v_{f i}$ was determined from Eq. (16) with $G$ being computed from Eq. (14). The integral of Eq. (14) was approximated by the trapezoidal rule. For convenience of application, it is worth to note that $b_{f i}=0$ and $h_{i} \rightarrow \infty, G$ approaches the effective capillary drive $\left(H_{c M}\right)$ defined by Morel-Seytoux and Khanji (1974):

$H_{c M}=\frac{1}{K_{s}} \int_{0}^{\infty} K d h$

When van Genuchten hydraulic models with the assumption of $m=1-1 / n$ are applied, $H_{c M}$ can be approximated as follows (Morel-Seytoux et al., 1996):

Table 1 - Van Genuchten parameters of 12 soils (Carsel and Parrish, 1988) used for the numerical studies.

\begin{tabular}{lccccc}
\hline Soil texture & $\theta_{r}$ & $\theta_{s}$ & $\alpha$ & $n$ & $K_{s}$ \\
\hline sand & & & $\mathrm{cm}^{-1}$ & & $\mathrm{~cm}$ per day \\
loamy sand & 0.045 & 0.43 & 0.145 & 2.68 & 712.8 \\
sandy loam & 0.057 & 0.41 & 0.124 & 2.28 & 350.2 \\
loam & 0.065 & 0.41 & 0.075 & 1.89 & 106.1 \\
silt & 0.034 & 0.46 & 0.016 & 1.37 & 6.00 \\
silt loam & 0.067 & 0.45 & 0.020 & 1.41 & 10.80 \\
sandy clay loam & 0.100 & 0.39 & 0.059 & 1.48 & 31.44 \\
clay loam & 0.095 & 0.41 & 0.019 & 1.31 & 6.24 \\
silty clay loam & 0.089 & 0.43 & 0.010 & 1.23 & 1.68 \\
sandy clay & 0.100 & 0.38 & 0.027 & 1.23 & 2.88 \\
silty clay & 0.070 & 0.36 & 0.005 & 1.09 & 0.48 \\
\hline clay & 0.068 & 0.38 & 0.008 & 1.09 & 4.80 \\
\hline
\end{tabular}


$H_{c M}=\frac{1}{\alpha} \frac{0.046 m+2.07 m^{2}+19.5 m^{3}}{1+4.7 m+16 m^{2}}$

with minimal errors $(<2 \%)$ over the range of $m$ from 0.05 to 0.7 . Eq. (21) was used to find a proper value for increments of $b$ in the approximation of $G$ using the trapezoidal rule. With $0.1 \mathrm{~cm}$ increments of $h$, approximations of the trapezoidal rule were close to those of Eq. (21).

To quantitatively evaluate the performance of the proposed scaling method, for each set of the scaled SWCPs, two criteria - the mean absolute error of scaling (MAES) and the root mean squared error of scaling of scaling (RMSES) - were defined as:

$M A E S=1 / N \sum_{i}^{n}\left|\theta_{i}^{*}-\theta_{m i}^{*}\right|$

RMSES $=\left[1 / N \sum_{i}^{n}\left(\theta_{i}^{*}-\theta_{m i}^{*}\right)^{2}\right]^{0.5}$ where $\theta_{m i}^{*}$ is the arithmetic mean of all $\theta^{*}$ values at each 0.1 increment of $z^{*}$ at which $\theta_{i}^{*}$ is located, and $N$ is the total number of the evaluated points. Profile of $\theta_{m}^{*}$ shows a mean scaled SWCPs, and therefore, MAES and RMSES indicate the deviations of the scaled SWCPs from the mean scaled SWCPs. When all the scaled SWCPs coalesce, these criteria will be equal to zero, suggesting an ideal performance of the scaling method.

\section{Results and Discussion}

Figure 2 shows HYDRUS model outputs for the 12 soils of Table 1 and initial conditions of A1 which were scaled by Eqs. (5), (6), and (10). Except for the sand, loamy sand and sandy loam, the remaining nine soils were reasonably well scaled and manifest a nearly unique scaled SWCP. A reason for the undesirable deviations in the three sandy soils is that the SWCPs in these soils with the imposed initial conditions are not similar in shape to those of the other soils. However, it seems that the main reason for the deviations is that, regarding the Nielsen-similarity condition, these soils are not similar to the other soils. To clarify this issue, the similarity condition should be evaluated.

As mentioned earlier, the so-called Nielsen-similarity deals with the shape similarity of the flux density curves during redistribution. It is assumed that the flux curves are described by the following power model (Jury and Horton, 2003):

Table 2 - Two sets of initial conditions (A and B) used for the numerical studies.

\begin{tabular}{lccccccccccccccc}
\hline \multicolumn{1}{c}{ Code } & A1 & A2 & A3 & A4 & A5 & A6 & B1 & B2 & B3 & B4 & B5 & B6 & B7 & B8 \\
\hline$z_{f i}(\mathrm{~cm})$ & 5 & 10 & 15 & 20 & 30 & 50 & 5 & 50 & 5 & 50 & 5 & 50 & 5 & 50 \\
$h_{f i}(\mathrm{~cm})$ & 10 & 8 & 6 & 4 & 2 & 0 & 10 & 10 & 0 & 0 & 10 & 10 & 0 & 0 \\
$h_{i}\left(\times 10^{4} \mathrm{~cm}\right)$ & 500 & 100 & 50 & 10 & 5 & 1 & 500 & 500 & 500 & 500 & 1 & 1 & 1 & 1 \\
\hline
\end{tabular}
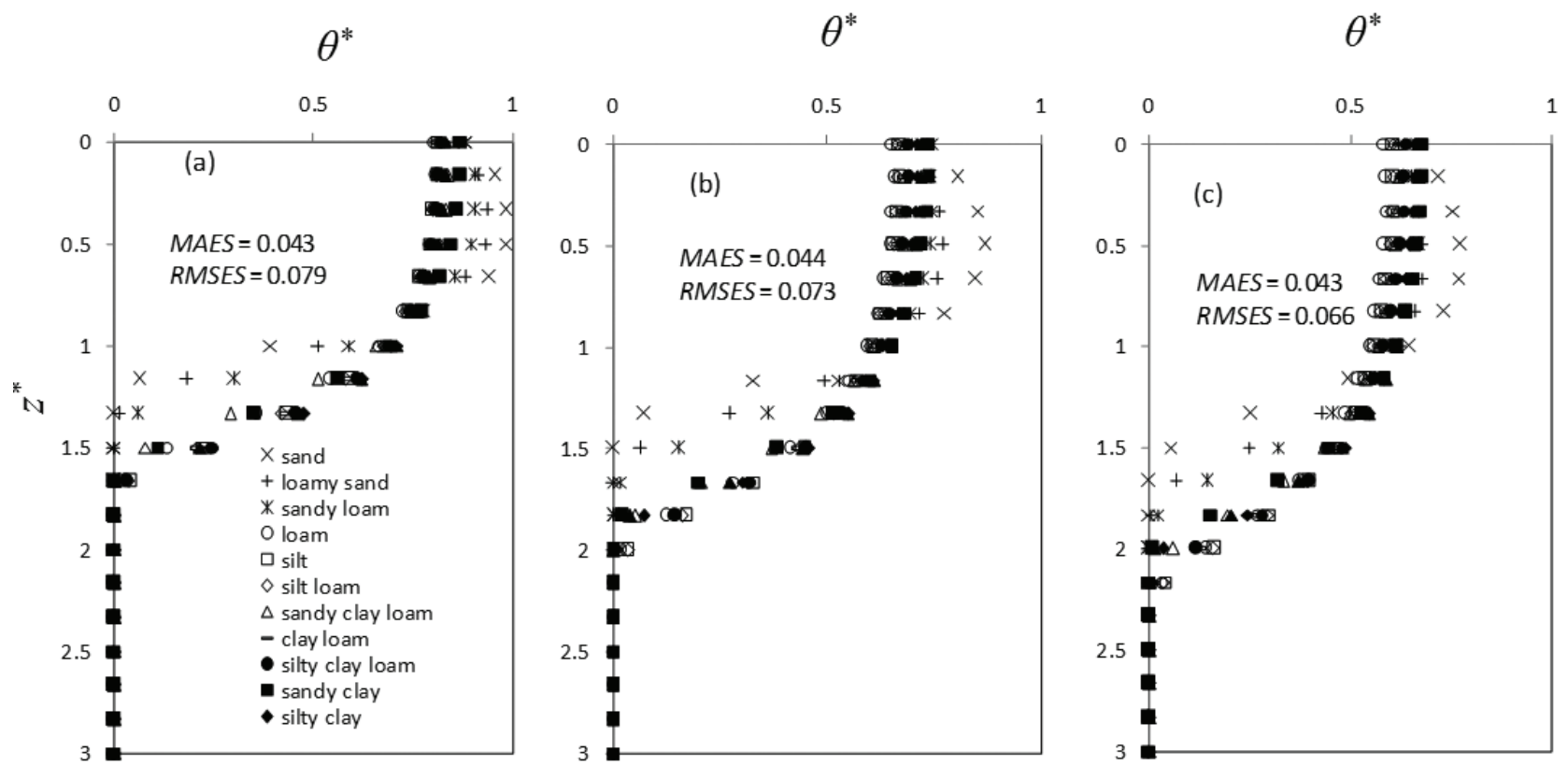

Figure 2 - Scaled soil water content profiles for the 12 soils of Carsel and Parrish (1988) (see Table 1) and initial conditions of A1 (see Table 2) at (a) $t^{*}=1$, (b) $t^{*}=5$, and (c) $t^{*}=10$.

Sci. Agric. (Piracicaba, Braz.), v.68, n.5, p.582-591, September/October 2011 
$q=a(t+b)^{c}$

where $a, b$, and $c$ are empirical fitting parameters. When time $t$ in Eq. (24) is longer than a day or so, the model shows a linear behavior. Therefore, the equality in the slope of the log-log plot of $q(t)$ verifies the similarity.

We studied the similarity condition in three soils of sand, loam, and clay textures under the conditions corresponding to Figure 2. For these soils, log-log transformed curves of $q(t)$ at $z=z_{f i}\left(z^{*}=1\right)$ are shown in Figure 3. The slope of the sand is definitely greater than those of the loam and clay which are nearly more equal (Figure 3). Therefore, we consider that the curves for the loam and clay are nearly similar but different from that of the sand.

Figure 4 shows the scaled SWCPs for the 12 soils and initial conditions of $\mathrm{A} 6$ which considers a deeply wetted initial profile. A comparison of Figures 2 and 4 indicates that by increasing $W$ (i.e. total water added to the soil profile) the scaling performance decreases. Since $d z_{f}^{*} / d t$ at $t=0$ is used as the scaling factor of time, the scaled SWCPs increasingly diverge as the time increases. The poor performance of the scaling in Figure 4 can be similarly justified by invalidity of the two similarity conditions required for scaling. For the sand, loam, and clay of Figure 4, log-log transformed curves of $q(t)$ at $z=Z_{f i}\left(z^{*}=1\right)$ are shown in Figure 5. The figure shows that the slopes are significantly different indicating that the Nielsen-similarity condition is not held in Figure 4. It can be concluded that by increasing $W$, the flow properties become more sensitive to soil texture. In other words, the textural range of the soils in which the similarity condition is held decreases as $W$ increases.

The impact of $W$ on the scaling performance, studied in detail by varying the initial conditions from A1 to A6, is shown in Figure 6 for the sand, loam, and clay at $t^{*}=5$.
We conclude that the scaling is more sensitive to $W$ for the very coarse- and very fine- textured soils. The scaled SWCPs for the loam are approximately invariant with respect to the initial conditions. For the sand, when $W$ is relatively small (A1 and A2), the scaled SWCPs show a delay, while the scaling is adequate for larger values of $W$. On the other hand, the scaled SWCPs in the clay coalesced for all initial conditions except for A6 - the condition corresponding to the greatest value of $W$.

To separately study the effects of $z_{f i}, b_{f i}$ and $b_{i}$ on the scaling performance, $\mathrm{RE}$ was solved by applying set $\mathrm{B}$ of the initial conditions in which, for $z_{f i}, h_{f i}$ and $h_{i}$, extreme values of set $\mathrm{A}$ are considered. The scaled solutions are presented in Figure 7 for sand, loam and clay at $t^{*}=5$. The figure indicates that, in

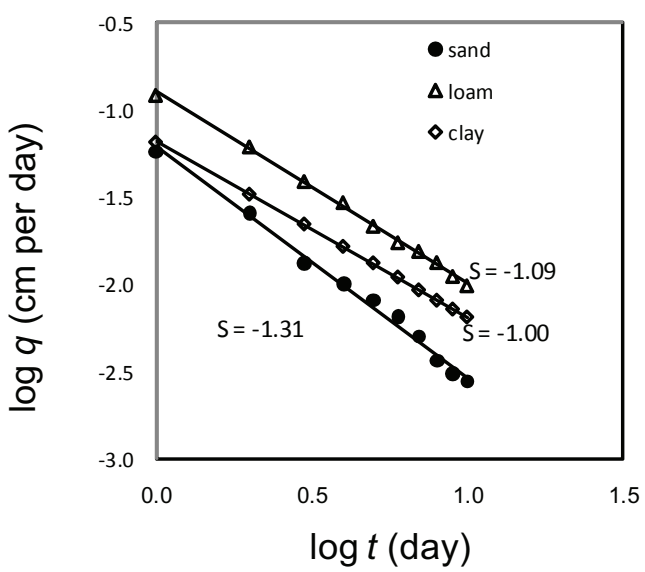

Figure 3 - Log-log transformed curves of the water flux density at $z=z_{f}\left(z^{*}=1\right)$ for three soils of sand, loam, and clay of Carsel and Parrish (1988) (see Table 1) under the conditions corresponding to Figure 2 . S represents the slope of the lines fitted to the points.
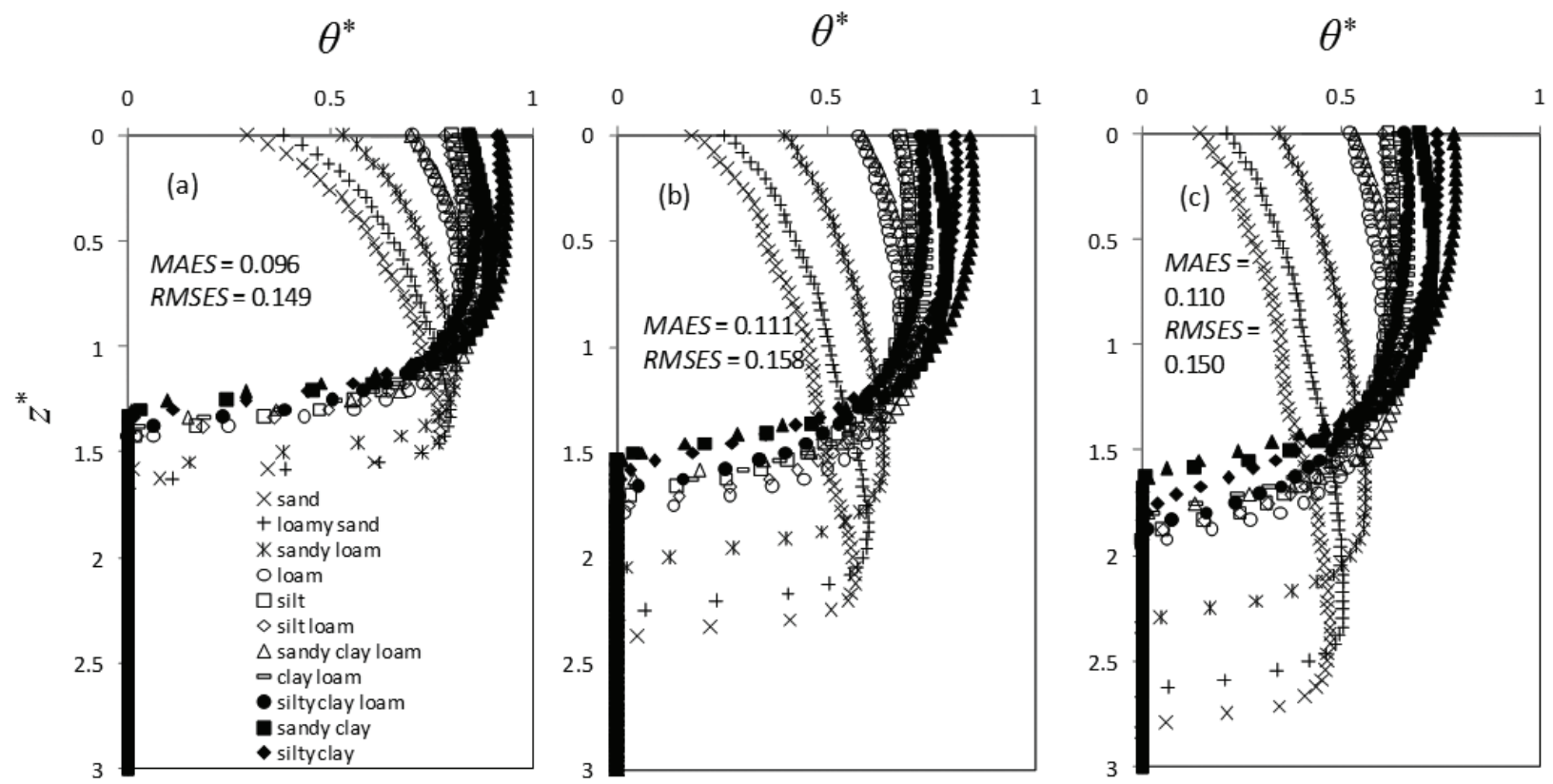

Figure 4 - Scaled soil water content profiles for the 12 soils of Carsel and Parrish (1988) (see Table 1) and initial conditions of A6 (see Table 2) at (a) $t^{*}=1$, (b) $t^{*}=5$, and (c) $t^{*}=10$. 
very coarse- or fine-textured soils, the scaling performance is more sensitive to $z_{f}, h_{f i}$ and $h_{i}$. The scaled SWCPs are approximately invariant for the loam while this is not the case for the sand and clay.

Impacts of $z_{f i}, h_{f i}$ and $h_{i}$ are dependent to each other. Based on the results, $h_{f i}$ seems to be the most effective parameter on the scaling performance especially for fine-textured soils. Variation of this parameter significantly changes the flow rate and therefore, the shape of the flux curves. Thus, cases with an extreme value of $h_{f i}$ will be far from the other cases regarding the Nielsen-similarity condition. Also, increasing $z_{f i}$ decreases the scaling performance (Figure 7). This is the case for the sand when $b_{f i}$ is large, however, for the clay when $b_{f i}$

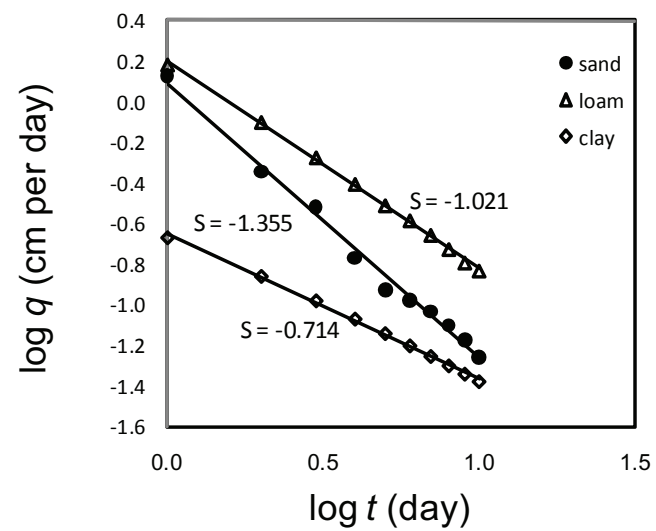

Figure 5 - Log-log transformed curves of the water flux density at $z=z_{f}\left(z^{*}=1\right)$ for three soils of sand, loam, and clay of Carsel and Parrish (1988) (see Table 1) under the conditions corresponding to Figure 4 . S represents the slope of the lines fitted to the points. is small. The results confirm that the flow properties are not significantly affected by variation of $b_{i}$. In the sand, the impact of $b_{i}$ is significant only if $h_{f i}$ is large. This is the case for the clay if simultaneously, $h_{f i}$ is large and $z_{f i}$ is small.

Generally, the above discussions suggest that the proposed scaling method can be successfully applied for medium- and fine-textured soils provided that the initial profile is not deeply wetted. Figure 8 shows the scaled SWCPs at three scaled times of 1,5 , and 10 for the nine medium- and fine-textured soils of Table 1 (i.e. from loam to clay) having initial conditions A1 through A5. Even though the figure contains 45 various scenarios for the solution of RE, the scaled SWCPs are nearly invariant with limited scattering around the mean scaled SWCPs (the white points in the figure).

The 45 cases considered in Figure 8 are approximately Nielsen-similar. For these 45 cases, Figure 9a shows the log-log transformed curves of $q(t)$ at $z=z_{f i}\left(z^{*}=1\right)$. It is obvious that the slopes are approximately equal with an average of -0.955 (standard deviation $=0.127$ ). Therefore, the flux curves could be well scaled using Eq. (17), which are presented in Figure 9b. Although the flux values fall in a wide range, Figure 9b indicates that the scaled fluxes coalesced into a unified curve and could be well described using a power model similar to Eq. (24), $q^{*}=0.71\left(t^{*}+0.069\right)^{-1.03}$, with a determination coefficient of 0.995. Recognizing that the two assumed similarity conditions are held for the range of soils and selected initial conditions, we propose that the scaling method can be generalized to numerous other cases in this range leading to approximations of the solutions of RE.

\section{Generalizing a Single Solution}

The ability of the scaling method to approximate numerical solutions of RE using a single solution was evaluated. To
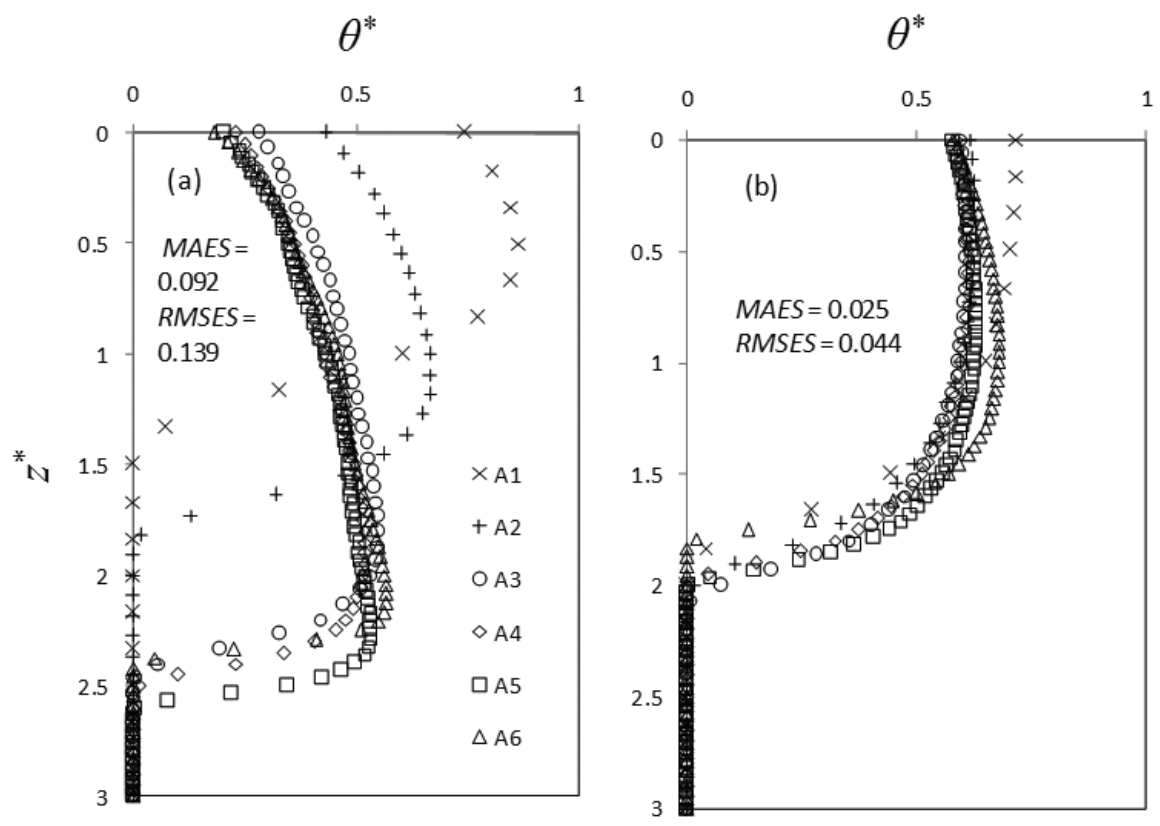

1

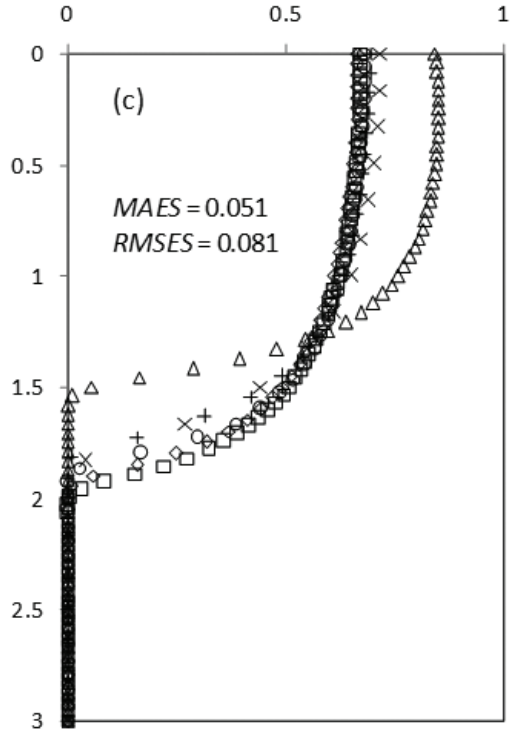

Figure 6 - Scaled soil water content profiles for (a) sand, (b) loam, and (c) clay of Carsel and Parrish (1988) (see Table 1) and set A of initial conditions (see Table 2) at $t^{*}=5$. 


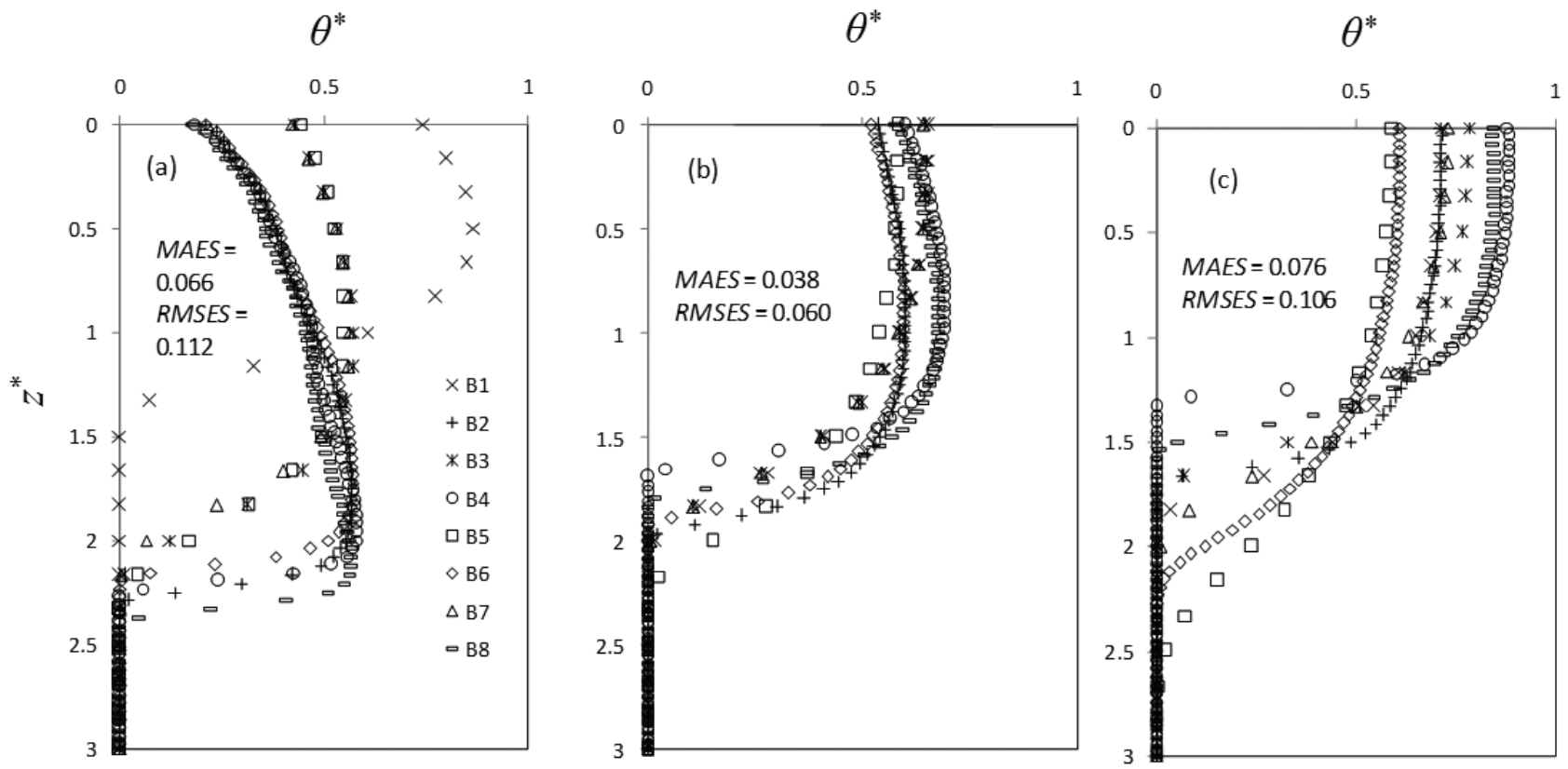

Figure 7 - Scaled soil water content profiles for (a) sand, (b) loam, and (c) clay of Carsel and Parrish (1988) (see Table 1) and set B of initial conditions (see Table 2) at $t^{*}=5$.

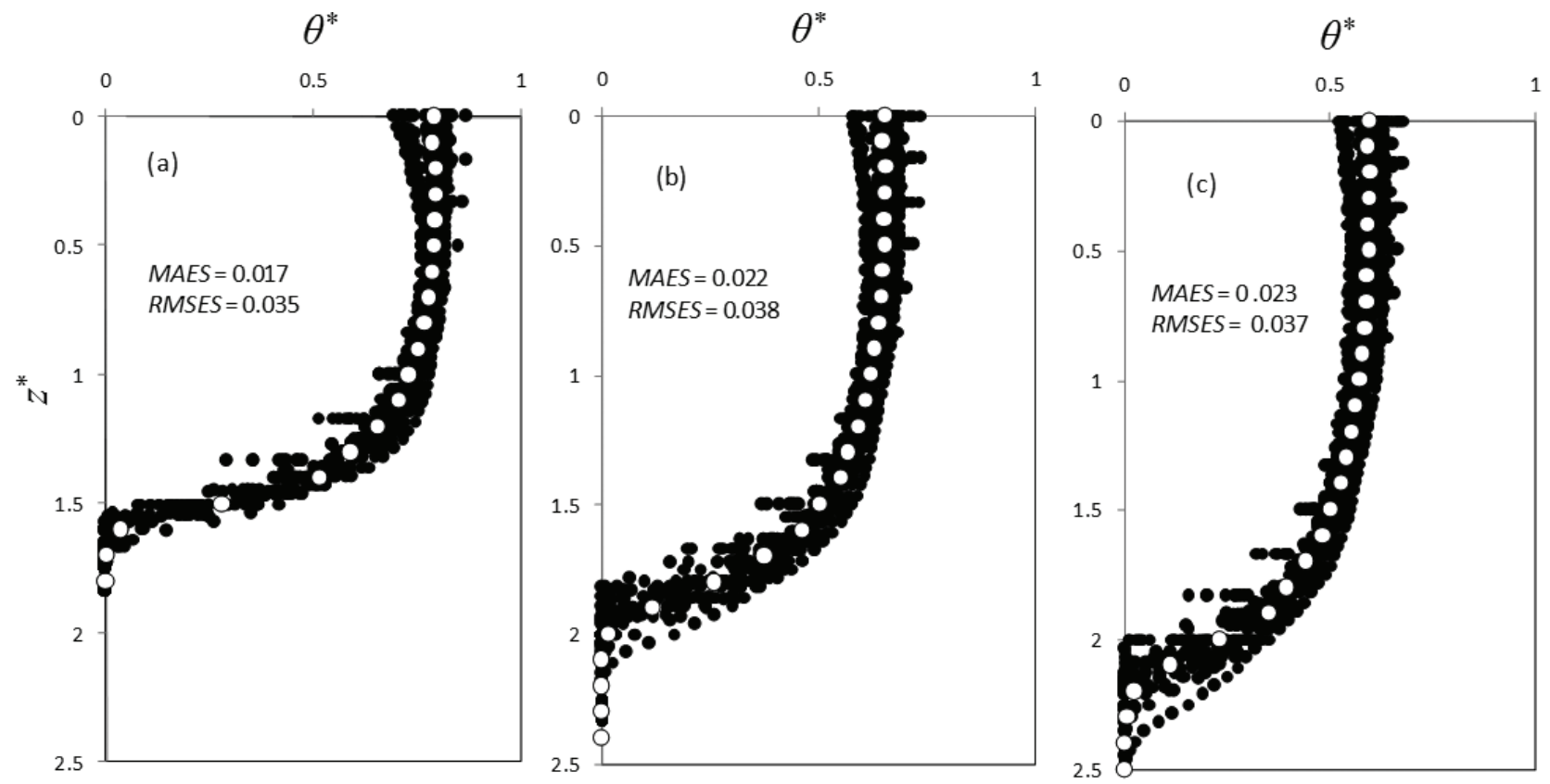

Figure 8 - Scaled soil water content profiles for medium- and fine-textured soils of Table 1 (from loam to clay) and set A of initial conditions except A6 (see Table 2) at (a) $t^{*}=1$, (b) $t^{*}=5$, and (c) $t^{*}=10$. White points show the mean scaled SWCPs.

do so, six medium- and fine-textured soils were selected from the literature: a Beit Netofa Clay (van Genuchten et al., 1980), a Pima Clay Loam (Elmaloglou and Malamos, 2003), and four other soils taken from UNSODA database (Leij et al., 1999) specified by codes $1300,1370,3360$, and 4030 . Some general information of these soils, including texture, taxonomic class and geographical location, are presented in le 3. For each soil, randomly produced initial conditions in the range of A1 to
A5 were considered. To determine the van Genuchten parameters of the four soils of UNSODA, van Genuchten hydraulic models, Eqs. (18) and (19), were simultaneously fitted to the measured data using the RETC software (van Genuchten et al., 1991). Table 4 presents the van Genuchten parameters of the soils as well as the initial conditions.

It was assumed that, for these six soils, the scaled solutions of RE are invariant and identical to the mean scaled SWCPs 

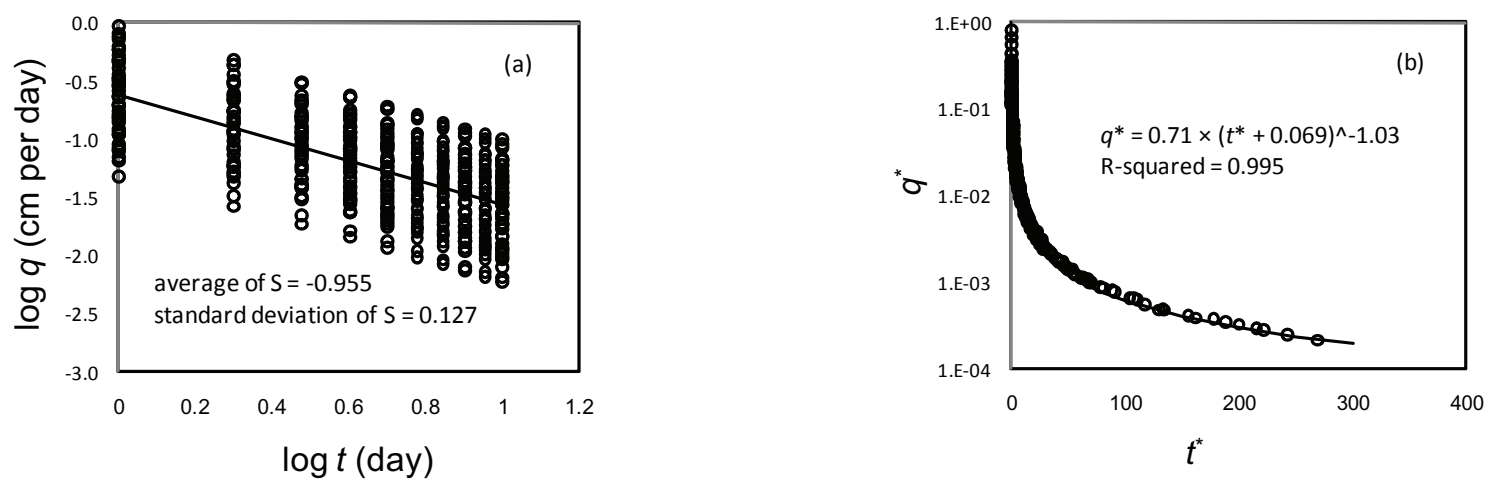

Figure 9 - (a) Unscaled and (b) scaled water flux density curves at $z=z_{f i}\left(z^{*}=1\right)$ for 45 cases of Figure 8. S represents the slope of the lines fitted to the points.

Table 3 - General information of the six selected soils: Beit Netofa Clay (van Genuchten, 1980), Pima Clay Loam (Elmaloglou and Malamos, 2003), and four other soils taken from UNSODA database (Leij et al., 1999).

\begin{tabular}{|c|c|c|c|}
\hline Soil name & Texture & Taxonomic class & Geographical location \\
\hline Beit Netofa Clay & clay & Rhodustalfs & $\begin{array}{l}\text { Beit Netofa valley, Lower Galilee, Israel }\left(32^{\circ} 44^{\prime} \mathrm{N}, 35^{\circ}\right. \\
\left.26^{\prime} \mathrm{E}\right)\end{array}$ \\
\hline Pima Clay Loam & clay laom & Cumulic Haplustoll & Marana, Arizona, USA ( $\left.32^{\circ} 24^{\prime} \mathrm{N}, 111^{\circ} 10^{\prime} \mathrm{W}\right)$ \\
\hline 1300 & sandy clay loam & Thermic Typic Torrifluvents & $\begin{array}{l}\text { Las Cruces, New Mexico, USA (USA - } 32^{\circ} 19^{\prime} \mathrm{N}, 106^{\circ} 45^{\prime} \\
\text { W) }\end{array}$ \\
\hline 1370 & loam & Gley-Pseudogley & Muenchehagen (Loccum), Germany (52²6’ N, 9¹1' E) \\
\hline 3360 & silt loam & Mesic Typic Hapludalf & Goettingen-Weende, Germany $\left(51^{\circ} 33^{\prime} \mathrm{N}, 9^{\circ} 55^{\prime} \mathrm{E}\right)$ \\
\hline 4030 & silt loam & Typic Hapludalf & Helecine (Leuven), Belgium( $\left.50^{\circ} 44^{\prime} \mathrm{N}, 4^{\circ} 41^{\prime} \mathrm{E}\right)$ \\
\hline
\end{tabular}

Table 4 -Van Genuchten parameters of the six selected soils: Beit Netofa Clay (Van Genuchten et al., 1980), Pima Clay Loam (Elmaloglou and Malamos, 2003), and four other soils taken from UNSODA database (Leij et al., 1999), as well as the randomly produced initial conditions.

\begin{tabular}{lcccccccc}
\hline Soil name & $\theta_{r}$ & $\theta_{s}$ & $\alpha$ & $n$ & $K_{s}$ & $z_{f i}$ & $h_{f i}$ & $h_{i}$ \\
\hline Beit Netofa Clay & 0.000 & 0.446 & 0.0015 & 1.17 & 0.08 & 22 & 8 & 2.49 \\
Pima Clay Loam & 0.200 & 0.550 & 0.0321 & 1.28 & 9.91 & 9 & 6 & 1.64 \\
1300 & 0.000 & 0.371 & 0.0225 & 1.26 & 9.59 & 20 & 7 & 1.31 \\
1370 & 0.190 & 0.608 & 0.0089 & 1.32 & 15.89 & 14 & 2 & 0.68 \\
3360 & 0.064 & 0.362 & 0.0062 & 1.39 & 2.08 & 18 & 6 & 1.31 \\
4030 & 0.000 & 0.415 & 0.0432 & 1.41 & 1.16 & 14 & 3 & 1.51 \\
\hline
\end{tabular}

of Figure 6. Subsequently, the mean scaled SWCPs were descaled (i.e. converted to the real scale) for the six soils using the Eqs. (5), (6) and (10). Hence, for each soil, SWCPs were approximated during redistribution. To evaluate the accuracy of the approximations, the SWCPs for the same soils and initial conditions were individually simulated using HYDRUS. The simulated and approximated SWCPs were compared using the mean relative error criterion, $\mathrm{MRE}$ (i.e. the mean of the absolute errors between the simulated and approximated values relative to the simulated values).

Figure 10 shows the simulated and approximated SWCPs for the six soils of Table 3 at two times corresponding to $t^{*}=1$ and $t^{*}=10$. With the MRE values never exceeding $5 \%$, the scaling method satisfactorily approximated the numerical solutions of $\mathrm{RE}$ for the selected soils and initial conditions.

\section{Conclusion}

Scaled soil water content profiles were found to be nearly invariant during scaled redistribution times for medium- to fine-textured soils when the initial profile was not deeply wetted. An advantage of this method is that it is not restricted to a specific soil hydraulic model. A disadvantage is that the method does not adequately scale water content redistribution profiles of sands and other coarse-textured soils wetted partially, or those of fine-textured soils wetted deeply. Textural range of the soils in which the similarity conditions are held decreases as the initial conditions deals with a deeply wetted profile. In such a condition, a classification of soils and initial conditions and then separately scaling of each class may alleviate the problem. The method is promising to reduce compli- 

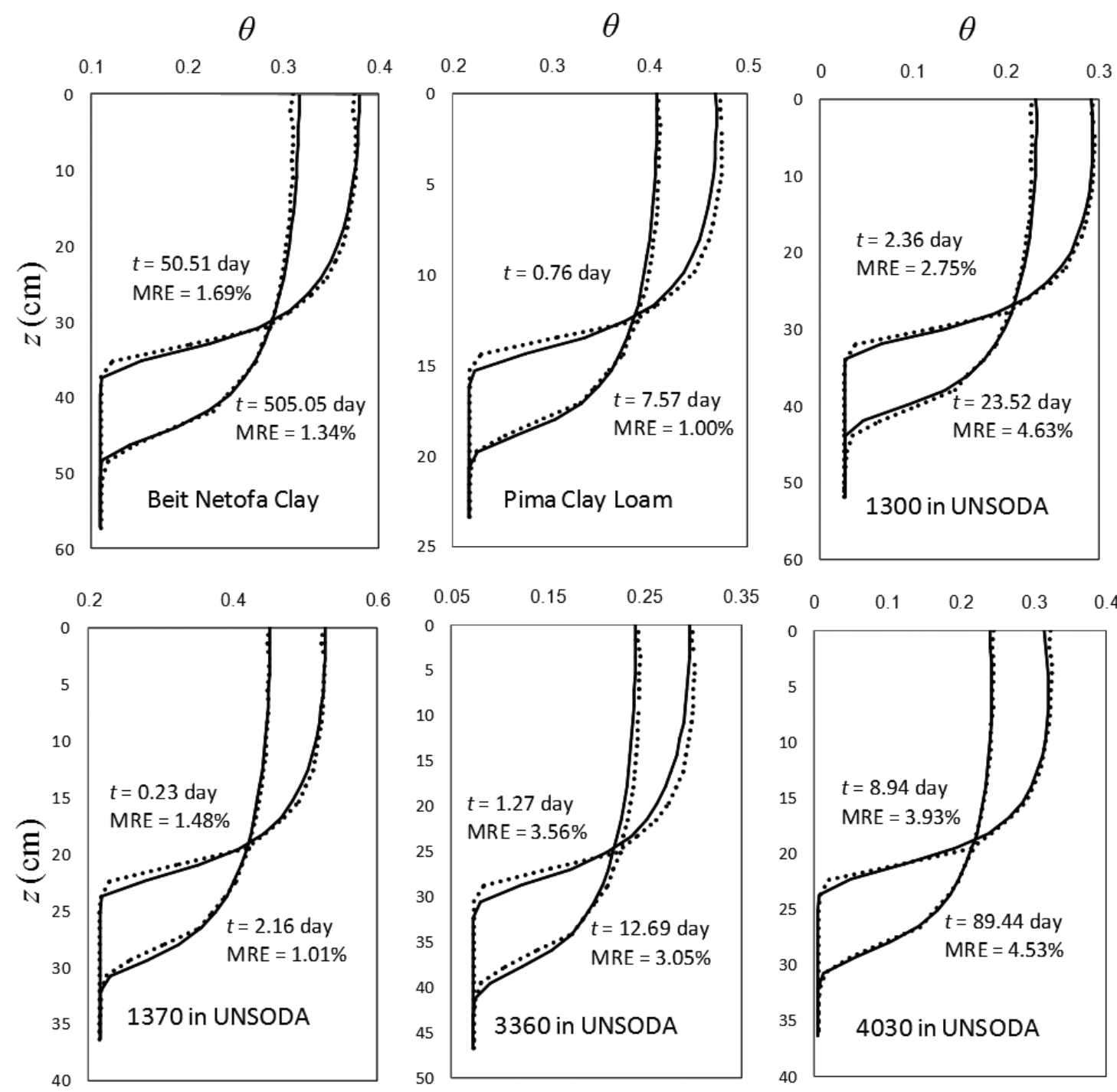

Figure 10 - Simulated (solid lines) and approximated (doted lines) soil water content profiles for the six selected soils: Beit Netofa Clay (van Genuchten et al., 1980), Pima Clay Loam (Elmaloglou and Malamos, 2003), and four other soils taken from UNSODA database (Leij et al., 1999) at two times corresponding to $t^{*}=1$ and $t^{*}=10$.

cated numerical calculations and opens a new window to easily obtain approximate solutions of highly nonlinear equation of Richards for water flow in unsaturated soils, within prescribed levels of error.

\section{Acknowledgement}

To Prof. D.R. Nielsen and Prof. A.W. Warrick, for their thorough review and helpful comments. The last author thanks $\mathrm{CNPq}$ for granting his productivity fellowship.

\section{References}

Ahuja, L.R.; Williams, R.D. 1991. Scaling water characteristic and hydraulic conductivity based on Gregson-Hector-McGowan approach. Soil Science Society of America Journal 55: 308-319.

Carsel, R.F.; Parrish, R.S. 1988. Developing joint probability distributions of soil water characteristics. Water Resources Research 24: 755-769.
Elmaloglou, S.T.; Malamos, N. 2003. A method to estimate soil water movement under a trickle surface line source, with water extraction by roots. Irrigation and Drainage 52: 273-284.

Jury, W.A.; Horton, R. 2003. Soil Physics. 6ed. John Wiley, New York, NY, USA.

Kozak, J.A.; Ahuja, L.R. 2005. Scaling of infiltration and redistribution of water across soil textural classes. Soil Science Society of America Journal 69: 816-827

Kosugi, K.; Hopmans, J.W. 1998. Scaling water retention curves for soils with lognormal pore-size distribution. Soil Science Society of America Journal 62: 1496-1504.

Kutilek, M.; Nielsen, D.R. 1994. Soil Hydrology. Catena, Germany.

Kutilek, M.; Zayani, K.; Haverkamp, R.; Parlange, J.Y.; Vachaud, G. 1991. Scaling of Richards' equation under invariant flux boundary conditions. Water Resources Research 27: 2181-2185.

Leij, F.J.; Alves, W.J.; van Genuchten, M.T.; Williams, J.R. 1999. The UNSODA unsaturated soil hydraulic database. p. 1269-1281. In: van Genuchten, M.T.; Leij, F.J.; Wu. L., eds. Characterization and measurement of the hydraulic properties of unsaturated porous media. University of California, Riverside, CA, USA.

Miller, E.E.; Miller, R.D. 1956. Physical theory for capillary flow phenomena. Journal of Applied Physics 27: 324-332. 
Morel-Seytoux, H.J.; Khanji, J. 1974. Derivation of an equation of infiltration. Water Resources Research 10: 795-800.

Morel-Seytoux, H.J.; Meyer, P.D.; Nachabe, M.; Touma, J; van Genuchten, M.T.; Lenhard, R.J. 1996. Parameter equivalence for the Brooks-Corey and van Genuchten soil characteristics: Preserving the effective capillary drive. Water Resources Research 32: 1251-1258.

Mualem, Y. 1976. A new model for predicting the hydraulic conductivity of unsaturated porous media. Water Resources Research 12: 513-22.

Neuweiler, I.; Cirpka, O.A. 2005. Homogenization of Richards equation in permeability fields with different connectivities. Water Resources Research 41: W02009, doi:10.1029/2004WR003329.

Reichardt, K.; Nielsen, D.R.; Biggar, J.W. 1972. Scaling of horizontal infiltration into homogeneous soils. Soil Science Society of America Proceedings 36: 241-245.

Roth, K. 2008. Scaling of water flow through porous media and soils. European Journal of Soil Science 59: 125-130.

Sadeghi, M.; Gohardous-Monfared, M.R.; Ghahraman, B. 2010. Scaling of soil hydraulic conductivity function using effective capillary drive. Journal of Water and Soil 24: 189-197 (in Persian).

Simmons, C.S.; Nielsen, D.R.; Biggar, J.W. 1979. Scaling of field-measured soil-water properties. Hilgardia 47: 77-173.

Simunek, J.; Sejna, M.; Saito, H.; Sakai, M.; van Genuchten, M.T. 2008. The HYDRUS-1D Software Package for Simulating the One-Dimensional Movement of Water, Heat, and Multiple Solutes in Variably-Saturated Media, Version 4.0. University of California, Riverside, CA, USA.

Sharma, M.L.; Gander, G.A.; Hunt, C.G. 1980. Spatial variability of infiltration in a watershed. Journal of Hydrology 45: 101-122.

Tuli, A.; Kosugi, K.; Hopmans, J.W. 2001. Simultaneous scaling of soil water retention and unsaturated hydraulic conductivity functions assuming lognormal pore-size distribution. Advance in Water Resources 24: 677-688.
Van Genuchten, M.T. 1980 A closed-form equation for predicting the hydraulic conductivity of unsaturated soils. Soil Science Society of America Journal 44: 892-898.

Van Genuchten, M.T.; Leij, F.J.; Yates, S.R. 1991. The RETC Code for Quantifying the Hydraulic Functions of Unsaturated Soils. Robert S. Kerr Environmental Research Laboratory, Ada, OK, USA.

Vereecken, H.; Kasteel, R.; Vanderborght, J.; Harter, T. 2007. Upscaling hydraulic properties and soil water flow processes in heterogeneous soils: a review. Vadose Zone Journal 6:1-28.

Vogel, T.; Cislerova, M.; Hopmans, J.W. 1991. Porous media with linearly hydraulic properties. Water Resources Research 27: 2735-2741.

Warrick, A.W.; Mullen, G.J.; Nielsen, D.R. 1977. Scaling of field measured hydraulic properties using a similar media concept. Water Resources Research 13: 355-362.

Warrick, A.W.; Amoozegar-Fard, A. 1979. Infiltration and drainage calculations using spatially scaled hydraulic properties. Water Resources Research 15: 1116-1120.

Warrick, A.W.; Lomen, D.O.; Yates, S.R. 1985. A generalized solution to infiltration. Soil Science Society of America Journal 49: 34-38.

Warrick, A.W.; Hussen, A.A. 1993. Scaling of Richards' equation for infiltration and drainage. Soil Science Society of America Journal 57: $15-18$.

Youngs, E.G. 1990. Application of scaling to soil-water movement considering hysteresis. p. 23-37. In: Hillel, D.; Elrick, D.E., eds. Scaling in soil physics: principles and applications. Soil Science Society of America, Madison, WI, USA.

$\overline{\text { Received July 22, } 2010}$

Accepted April 20, 2011 